\title{
Affective analysis of customer service calls
}

\author{
Vera Cabarrão ${ }^{1,2}$, Mariana Julião ${ }^{1,3}$, Rubén Solera-Ureña ${ }^{1}$, Helena \\ Moniz $^{1,2,4}$, Fernando Batista ${ }^{1,5}$, Isabel Trancoso ${ }^{1,3}$, Ana Isabel Mata ${ }^{2}$ \\ ${ }^{1}$ Laboratório de Sistemas de Língua Falada, INESC-ID Lisboa, Portugal \\ ${ }^{2}$ FLUL/CLUL, Universidade de Lisboa, Portugal \\ ${ }^{3}$ Instituto Superior Técnico, Universidade de Lisboa, Portugal \\ ${ }^{4}$ Unbabel Lda., Portugal \\ ${ }^{5}$ Instituto Universitário de Lisboa (ISCTE-IUL), Portugal \\ https://doi.org/10.36505/ExLing-2019/10/0009/000371
}

\begin{abstract}
This paper presents an affective and acoustic-prosodic analysis of a call-center corpus (700 phone calls with corresponding customer satisfaction levels). Our main goal is to understand how customers' satisfaction correlates to the acoustic-prosodic and affective information (emotions and personality traits) of the interactions. A subset of 30 calls was manually annotated with emotions (frustrated vs. neutral) and personality traits (Big-Five model). Results on automatic satisfaction prediction from acousticprosodic features show a number of very informative linguistic knowledge-based features, especially pitch and energy ranges. The affective analysis also provides encouraging results, relating low/high satisfaction levels with the presence/absence of customer frustration. Concerning personality, customers tend to express signs of anxiety and nervousness, while agents are generally perceived as extroverted and open.
\end{abstract}

Key words: acoustic-prosodic features, emotions, personality traits, call-center interactions, customer satisfaction

\section{Introduction}

Customer support is essential to assuring the bonds between customers and service providers, and for maintaining frequent contacts and offering solutions to customers' problems. Measuring customers' satisfaction is necessary to distinguish between satisfied and loyal clients vs. upset ones who will most probably subscribe to services in other companies. Customers' satisfaction correlation with linguistic features (lexical, acousticprosodic) and paralinguistic events (e.g., emotions, personality traits) in customer-agent interactions can help companies to develop automatic procedures to understand whether the customer is satisfied with the service or not, and how the agent performed to achieve a successful outcome. Literature on customer satisfaction (e.g., Lotko, 2015; Delcourt et al., 2013; Stoyanchev, Maiti and Bangalore, 2019), and emotions detection (e.g., Lee and Narayanan, 2005; Vidrascu and Devillers, 2007) has shown diverse findings on how agents interact with customers successfully. Our main goal is to understand how customers' satisfaction level (satisfied vs. nonsatisfied) correlates with the acoustic-prosodic clues extracted

ExLing 2019: Proceedings of $10^{\text {th }}$ International Conference of Experimental Linguistics, 25-27 September 2019, Lisbon, Portugal 
from speech interactions in a European Portuguese (EP) call-center corpus, as well as with emotions and personality traits pertained to either the customer or the agent. To the best of our knowledge, this is the first study on speech-based affective computing for customer satisfaction in EP.

\section{Methodology}

The CCS (call-center satisfaction) corpus comprises around 700 call-center phone calls between customers and agents. The conversations were collected from a non-technical support service in Portugal. All the calls are labelled according to the Net Promoter Score (NPS, Reichheld, 2003), a metric used to express customers' satisfaction. NPS scores were provided by the customers at the end of the calls according to their overall satisfaction with the service and their hypothetical leaning towards recommending it (promoters) or not (detractors) to their friends and family.

This corpus was divided into train, development, and test subsets, and was used for creating a machine learning model for automatic satisfaction prediction. In addition, an expert annotator labelled a subset of 30 calls. Customers' and agents' speech turns were annotated in terms of emotions as either neutral or frustrated, and their overall personality was annotated according to the Big-Five traits: openness (artistic, imaginative), conscientiousness (organized, efficient), extroversion (outgoing, talkative), agreeableness (kind, sympathetic), and neuroticism (anxious, worrying).

The experiments here reported use two different sets of acousticprosodic features typically adopted in computational paralinguistic tasks, namely the feature set used in Schuller et al. (2012) and the eGeMAPS set (Eyben et al, 2015), and also a set of knowledge-based features (KB) that take into account linguistic information at the intra and inter-pausal unit levels (IPUs).

\section{Results}

The manual annotation of emotions shows that "neutral" is the most common emotion in both high and low customers' satisfaction groups. This was expected, given the nature of the corpus: in most phone calls, clients aim to solve small problems, and do not tend to present an aggressive posture towards the agent. "Frustration" is mainly associated with customers and usually decreases along the call. The comparison between detractors and promoters does not reveal a significant difference in "frustration" for both groups $(22.5 \%$ vs. $16.3 \%$, respectively). Therefore, we can hypothesize that, even though frustrated clients are present in both satisfaction groups, the performance of the agent may influence the customers' evaluations towards a high or a low score according to the interactions between them.

For personality traits, the manual annotation of the corpus shows that the most salient traits for the agents in the low satisfaction group are conscientiousness (all the agents), agreeableness (83\%), and extroversion (67\%). 
No agent in this group was labelled as neurotic, as opposed to the users (all users were associated with this trait). Clients were also labelled as conscientious (83\%). These results show that the most distinctive traits between agents and users in this group are neuroticism and openness (no users were associated with the openness trait). In the group with high satisfaction level, neuroticism and openness are also the most distinctive traits between clients and agents: 5\% of the agents were labelled as neurotic vs. $73 \%$ of the users, and $83 \%$ of agents as open vs. $28 \%$ of users. For the remaining traits, they tend to be similar.

Concerning the automatic prediction of the satisfaction levels based on acoustic-prosodic cues, the model (a linear SVM) that uses the linguistic KBfeatures (41 features only) achieves a satisfactory performance. These features are very informative (unweighted average recall -UAR- of $75.4 \%$ ), with a better recall for non-satisfied $(81 \%)$ than for satisfied $(68 \%)$ users. Combined with the eGeMAPS features, the model improves the recall for satisfied users to $75 \%$. We also carried out a relevance analysis of the KBfeatures based on their corresponding weights in the SVM classifier. Pitch range and dynamics play a major role in the prediction of satisfaction. A possible explanation for these results is related to the linguistic KB-features focusing on dynamics between inter-pausal units (IPUs), which can be related with the customer-agent interactions changing throughout the call. Energy dynamics and range are also informative, helping for distinguishing between satisfied and non-satisfied clients.

\section{Conclusions}

Customer support analytics has evolved due to the impact of affective computing research. The subjective assessment of customer satisfaction is being further supported by objective and quantifiable measures of emotions and other social human behaviors expressed in speech.

Emotions are crucial to better understand the dynamics of customer-agent interactions. Our results show that neutral and frustrated speech segments are transversal in the corpus, but frustrated segments in the promoters group appear usually at the beginning of the call, changing their dynamics influenced by the agent's behavior throughout the conversation. Personality traits do not seem to have a great impact on the satisfaction level discrimination, but are crucial to distinguish customers from agents. To this respect, customers are mainly described as nervous and anxious (neurotic) while agents are perceived as conscious, open and extroverted. Regarding the automatic prediction of the satisfaction levels based on acoustic-prosodic clues, our models achieved satisfactory performances. Results show that the linguistic KB-features are very informative (UAR of $75.4 \%$ ), with a better recall for non-satisfied users $(81 \%)$ than for satisfied. The most informative features are pitch and energy range and dynamics between IPUs, which can be associated with customer-agent interactions changing throughout the call. Although these are preliminary 
40 V. Cabarrão et al.

results due to the size of the corpus, our results may still be regarded as encouraging.

\section{Acknowledgements}

This work was supported by national funds through Fundação para a Ciência e a Tecnologia (FCT) with references UID/CEC/50021/2019 and UID/LIN/00214/2019, and under PhD grants SFRH/BD/96492/2013 and SFRH/BD/139473/2018.

\section{References}

Delcourt, C., Gremler, D.D., Van Riel, A.C. and Van Birgelen, M., 2013. Effects of perceived employee emotional competence on customer satisfaction and loyalty: The mediating role of rapport. Journal of Service Management, 24(1), 5-24.

Eyben, F., Scherer, K.R., Schuller, B.W., Sundberg, J., André, E., Busso, C., Devillers, L.Y., Epps, J., Laukka, P., Narayanan, S.S. and Truong, K.P., 2015. The Geneva Minimalistic Acoustic Parameter Set (GeMAPS) for Voice Research and Affective Computing. IEEE Transactions on Affective Computing, 7(2), 190-202.

Lee, C.M. and Narayanan, S.S., 2005. Toward detecting emotions in spoken dialogs. IEEE Transactions on Speech and Audio Processing, 13(2), 293-303.

Lotko, A., 2015. Classifying customers according to NPS index: cluster analysis for contact center services. Central European Review of Economics \&

Finance, 8(2), 5-30.

Reichheld, F.F., 2003. The one number you need to grow. Harvard Business Review, 81(12), 46-55.

Schuller, B.W., Steidl, S., Batliner, A., Nöth, E., Vinciarelli, A. and Burkhardt, F., 2012. The INTERSPEECH 2012 Speaker Trait Challenge. Proc. of Interspeech 2012, Portland, OR, USA.

Stoyanchev, S., Maiti, S. and Bangalore, S., 2019. Predicting interaction quality in customer service dialogs. In Advanced Social Interaction with Agents, 149-159. Springer, Cham.

Vidrascu, L. and Devillers, L., 2007. Five emotion classes detection in real-world call center data: the use of various types of paralinguistic features. Proc. of International Workshop on Paralinguistic Speech between Models and Data, Saarbrücken, Germany. 